\title{
Cake filtration mechanisms in steady and unsteady flows
}

\author{
M.R. Mackley and N.E. Sherman \\ Department of Chemical Engineering, University of Cambridge, Pembroke Street, Cambridge CB2 3RA (UK)
}

(Received April 29, 1992; accepted in revised form October 21, 1992)

\begin{abstract}
The cake filtration kinetics of a neutrally buoyant suspension of $\sim 150 \mu$ m diameter polyethylene particles have been studied in laminar and oscillatory flows. Simultaneous measurements of both filtrate flux and cake height have been made, together with direct in situ optical observations of particle behaviour at the cake-slurry interface. Mechanisms for the cake formation process have been identified, and under steady crossflow conditions, regimes of selective packing and decreasing particle capture probability have been observed. The effect on the filtration mechanisms of unsteadiness in the crossflow has been investigated. Sinusoidal oscillations have been imposed upon a constant net flow in a channel geometry and the effect of the introduction of baffles above the filter medium has been examined. Flow patterns showing the behaviour of particles in the vicinity of the filter have been obtained which show that eddy circulation, both upstream and downstream of the baffles, can cause filter cake erosion. This effect has been enhanced by periodic switching of the differential pressure across the filter cake and its applicability for filtrate flux improvement has been investigated.
\end{abstract}

Keywords: cake filtration; cake formation; fouling

\section{Introduction}

Crossflow filtration is employed in many phase separation processes, for example the dewatering of mineral slurries [1], harvesting of plant and animal cells [2] and hemofiltration [3]. The behaviour generally observed for these processes is that initially the fluid out-flow declines rapidly as a filter cake is formed, but as the crossflow limits the cake growth a limiting or dynamic regime is approached. Clearly it is desirable to limit the filter cake growth and it would appear that any fluid mechanical scheme that inhibits cake build up should enhance the

Correspondence to: N.E. Sherman, University of Cambridge, Pembroke Street, Cambridge CB2 3RA, UK. filtrate flux. Indeed, it has been demonstrated that oscillatory crossflow in the presence of baffles provides such a scheme [4]. In order to investigate this, we have examined the filtration behaviour of a neutrally buoyant $\sim 150 \mu \mathrm{m}$ size polyethylene particle suspension, under a range of applied crossflow conditions. These particles scatter light strongly and have enabled us to obtain direct in situ observations of cake formation together with simultaneous measurements of both the cake height and filtrate flux.

\section{Experimental}

A model suspension of neutrally buoyant polyethylene particles, having diameters in the 
range 125 to $180 \mu \mathrm{m}$, dispersed in a Newtonian liquid comprising water and methylated spirit (viscosity $=2.38 \mathrm{mPa}-\mathrm{sec}$ at $22^{\circ} \mathrm{C}$ ) was prepared at a solids volume concentration of $0.05 \%$. The experimental filtration rig used for this suspension is described in more detail elsewhere [5]. The essential features were that (i) the flow channel geometry provided plane Poiseuille steady flow above the filter and facilitated cake height observations, (ii) simultaneously with cake height measurements, filtrate flux was continuously monitored, (iii) the filtration could be conducted under constant differential pressure conditions of $5 \mathrm{kPa}$, being much greater than the pressure drop along the filter channel, i.e. $\sim 0.1 \mathrm{kPa}$, (iv) an optical resolution of $\sim 100 \mu \mathrm{m}$ was achieved such that individual particles could be readily observed.

The suspension was filtered under crossflow conditions for channel mean velocities $(\bar{v})$ in the range 0 to $0.25 \mathrm{~m} / \mathrm{sec}$. Defining the net flow Reynolds number,

$R e_{\mathrm{n}}=(\bar{v} H \rho) / \eta$

where $H$ is the channel height and $\rho, \eta$ are the fluid density and viscosity respectively, this $\bar{v}$ range corresponds to $R e_{\mathrm{n}} \sim 0$ to 950 . Further, at a constant $R e_{\mathrm{n}}=270$, cake formation observations and filtrate flux measurements were made under conditions where sinusoidal oscillations were imposed upon the crossflow. Also the effect of the inclusion of wall and centre baffles in the filter section was examined. The experimental geometry, shown in Fig. 1, was chosen for near-optimum inter-baffle fluid mixing with a baffle area constriction of $50 \%$ and a baffle spacing of 1.5 times $H$ as found by Brunold et al. [6]. These flows can be characterised in terms of an oscillatory Reynolds number,

$R e_{\mathrm{o}}=\left(\omega x_{0} H \rho\right) / \eta$

and the Strouhal number, a.

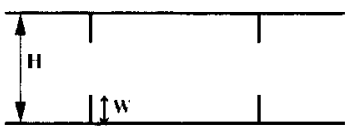

b.

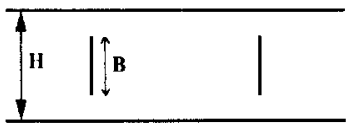

Fig. 1. The baffled channel. (a) Wall baffles, (b) centre baffles. $H=10 \mathrm{~mm}, W=2.5 \mathrm{~mm}$ and $B=5 \mathrm{~mm}$.

$S t=H /\left(2 \pi x_{0}\right)$

where $\omega$ is the angular frequency of oscillation and $x_{0}$ is the centre to peak amplitude of oscillation. Given that $2 x_{0}$ was set at $14.5 \mathrm{~mm}$ and that the frequency of oscillation was $\sim 1.7 \mathrm{~Hz}$, the experiments were conducted at $S t=0.22$ and $R e_{\mathrm{o}}=300$ (c.f. $R e_{\mathrm{n}}=270$ ). Finally the efficiency of cake erosion by these flows was investigated by employing periodic switching of the differential pressure driving the filtration process.

\section{Results and discussion}

\section{(a) Steady flow}

\section{Kinetics}

The data obtained for cake height and filtrate flux as a function of time under steady crossflow conditions are shown in Figs. 2 and 3 respectively. It can be seen in Fig. 2 that the effect of the application of crossflow is to limit cake growth. In Fig. 3, the flux exhibits an initial rapid decline followed by a levelling off towards a constant value. This is consistent with the observations of limiting cake height shown in Fig. 2. However, thinner cakes are formed for higher crossflow values but surprisingly the limiting filtrate flux decreases with increasing crossflow. This indicates that the thinner cakes produced by higher crossflow velocities offer more filtration resistance than thicker ones formed at lower velocities. This effect was found to be very reproducible and the data shown rep- 


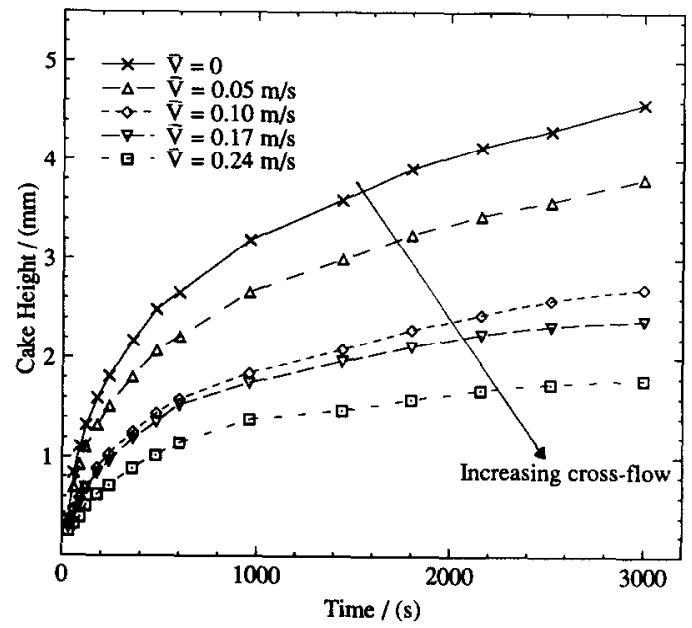

Fig. 2. Cake height vs. time.

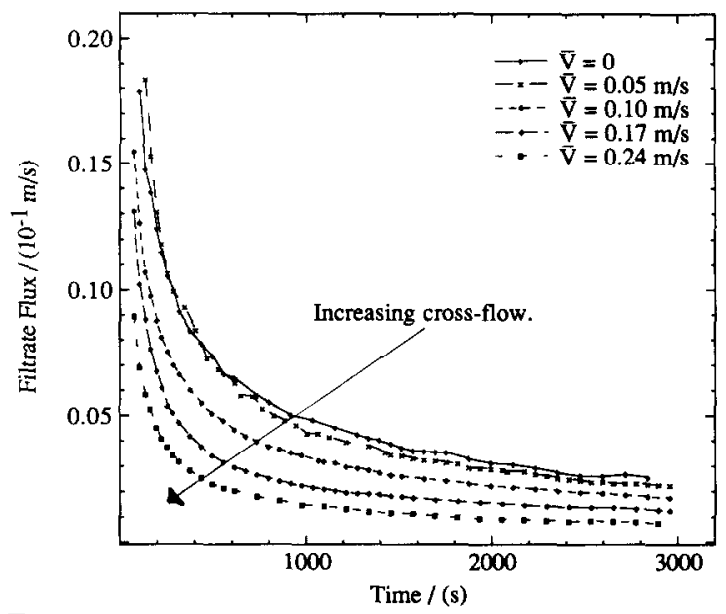

Fig. 3. Filtrate flux vs. time.

resents an average over ten operations under each set of experimental conditions.

Direct in situ observations of cake growth

Evidence for the effect of crossflow on particle packing has been found in the flow visualisation of cake formation. This showed that particles were drawn towards the filter cake in a manner determined by the relative magnitudes of the crossflow velocity and the filtrate flux. At early times when the wall flux domi- nated, particles approached the cake at very steep angles and attached irreversibly to the cake at the site of impact. In this case packing was non-selective. At later times when the crossflow dominated, particles approached the cake at very shallow angles and after impact they were observed to roll along the cake until captured at an energetically stable surface site. Eventually a regime was reached where particles impacting upon the cake did not necessarily attach to it. In this case the particle packing was highly selective. From these observations a mathematical filtration model based upon selective particle packing and surface capture has been developed, which is consistent with the experimentally measured filtrate flux and cake height [5].

\section{(b) Oscillatory flow}

\section{Kinetics}

The data obtained for filtration rate as a function of time using oscillatory crossflow with wall and centre baffles are shown in Figs. 4 and 5 respectively. It can be seen that oscillations or baffles alone do not improve the long term flux compared to the base case of steady crossflow. However in both cases the combination of

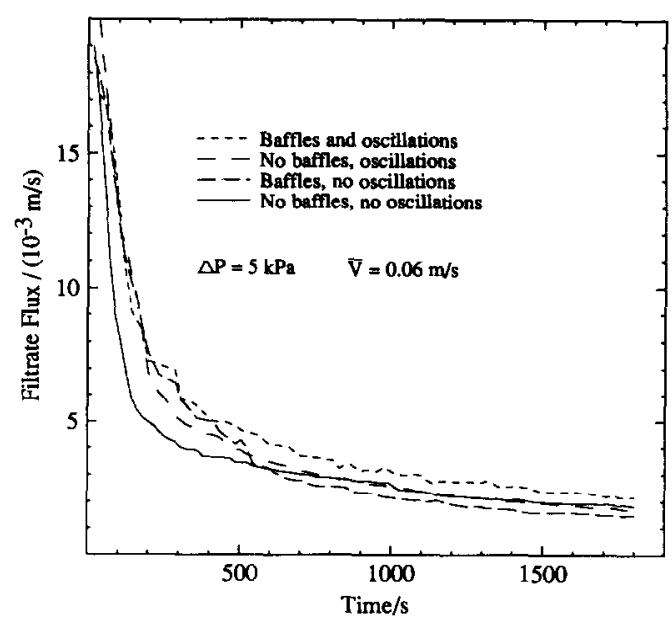

Fig. 4. Filtration rate vs. time. Wall baffle geometry. 


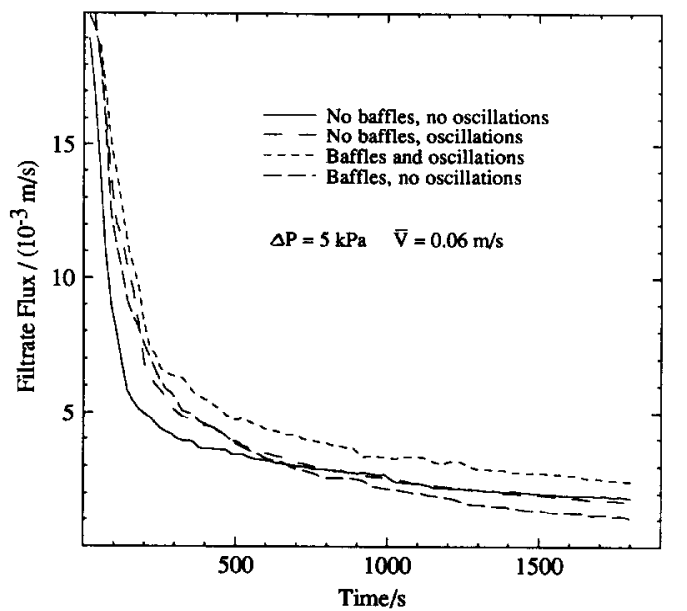

Fig. 5. Filtration rate vs. time. Centre baffle geometry.

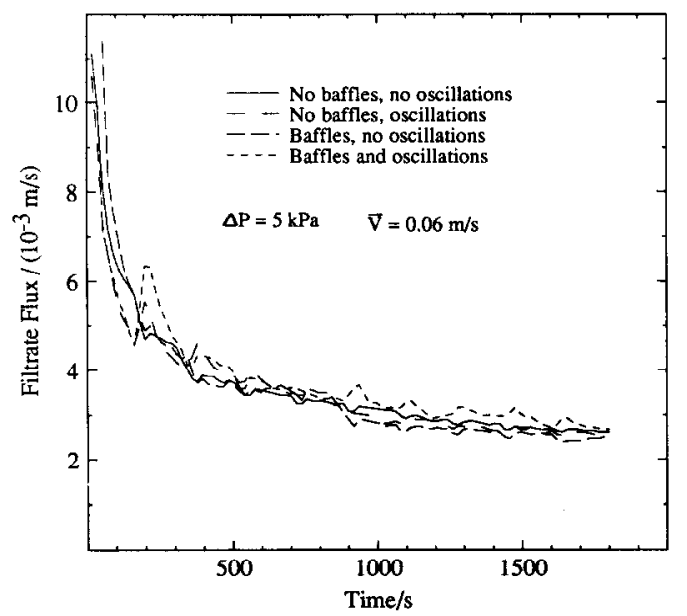

Fig. 6. Filtration rate vs. time. Wall baffles and differential pressure switching.

oscillatory flow and baffles does lead to a small enhancement of the limiting filtrate flux, with the centre baffle geometry being most effective giving an approximately $30 \%$ increase.

The data in Figs. 6 and 7 shows the effect on these flux kinetics of periodic differential pressure switching across the cake. In each case the filtrate outlet valve has been closed for $2 \mathrm{sec}$ every $180 \mathrm{sec}$ to allow erosion of the filter cake by the crossflow fluid stream. In the case of Fig. 6 , it can be seen that the inclusion of wall baf-

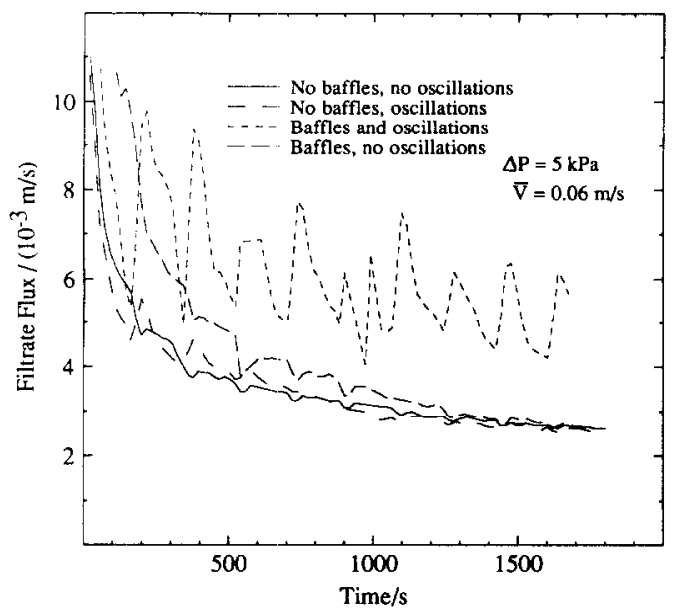

Fig. 7. Filtration rate vs. time. Centre baffles and differential pressure switching.

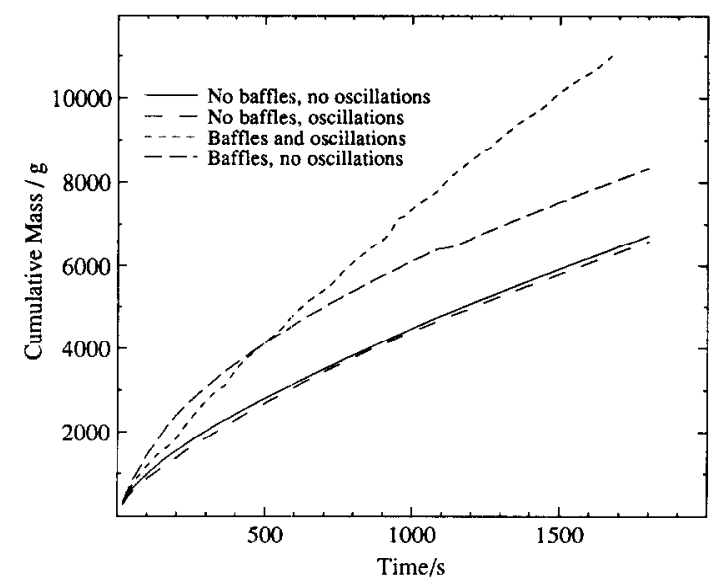

Fig. 8. Cumulative mass vs. time.

fles and/or flow oscillations does not have a profound effect on the long term flux, although there is a marginal increase above the baseline steady crossflow case when both baffles and oscillations are present. However the data in Fig. 7 shows that when centre baffles and flow oscillations are employed, the filtrate flux is substantially increased being approximately double that found in steady flow. The effect is clearly demonstrated in Fig. 8 where the cumulative mass of filtrate is plotted as a function of time. Initially the presence of baffles 
alone has the greatest effect, as the initial particle deposition is impeded due to enhanced tangential motion. However, in the long term the increase in mass with time for the case where both baffles and oscillations are present, is approximately double that found for the other conditions.

\section{Direct in situ observations of particles}

An explanation for the considerable flux enhancement obtained has been found from the identification of the cake erosion mechanisms for the various fluid mechanical conditions. It was seen that during the period when the filtrate outlet valve is closed (i.e. the differential pressure is switched off), the efficiency of particle removal from the cake was much greater in the case of oscillatory flow with centre baffles than for any other case.

Under steady flow conditions the particles at the cake surface were observed to flow tangentially across the cake and be re-entrained into the fluid flow. The imposition of oscillations was seen to impede this erosion in that the net tangential movement of particles decreased, although the unsteadiness in the flow did produce particle re-entrainment at the leading edge of the cake. When wall baffles were placed in the steady net flow, recirculating eddies formed downstream of the baffle tips producing some radial particle motion. However the cake was also shielded from the crossflow as it lay in the wake of the baffle and the consequent net removal of particles was similar to that observed for steady flow alone. The effect of the imposition of oscillations in this case was to alternately produce eddies upstream and downstream of the baffles, again having similar net erosion effect as the base case. These observations are consistent with the corresponding filtrate flux data shown in Fig. 6, where the differences observed are marginal.

With steady net flow in the presence of centre baffles, the acceleration of the fluid around the baffle tips provided a slight localised enhancement of the tangential particle motion on the cake surface (and hence cake erosion) as compared to the case of steady flow alone. This is consistent with the enhanced flux observed at early times in Fig. 7, although the long term value settles down close to the base case. When flow oscillations are imposed in the centre baffle geometry, eddies are formed alternatively upstream and downstream of the baffles. This situation is shown in Fig. 9 where the dotted line marks the position of the filter and $H$ is the channel height. The sequence in column (a) starting from the top, shows the fluid initially accelerating from left to right (the net flow direction) and then decelerating on progression to the point of flow reversal. It can be seen that eddies formed downstream of the baffles on the forward stroke eject particles from the cake into the fluid stream. As the flow reverses (Fig. 9 column b) eddies are formed upstream of the baffles, producing further re-entrainment of particles which are then swept away into the bulk stream with the net flow. The erosion efficiency is dramatically increased and consequently the corresponding filtrate flux is enhanced as seen in Fig. 7 .

Although the wall baffle geometry was not found to be as efficient as the centre baffles for cake erosion, it was found that qualitatively similar particle re-entrainment could be obtained if the filtrate outlet valve was closed for a longer period (e.g. $4 \mathrm{sec}$ instead of $2 \mathrm{sec}$ ) or if the crossflow velocity was increased. Figure 10 shows the observed flow patterns for the wall baffle case when $R e_{n}$ has been increased from 270 to 600 . The sequence in column (a) starts at the point of flow reversal and shows particles being ejected of the cake from behind the baffles into the central fluid stream. As the flow accelerates from left to right, in column (b), material is drawn into the crossflow by eddies which are shed downstream of the baffles and roll along the cake surface. This ejection of par- 


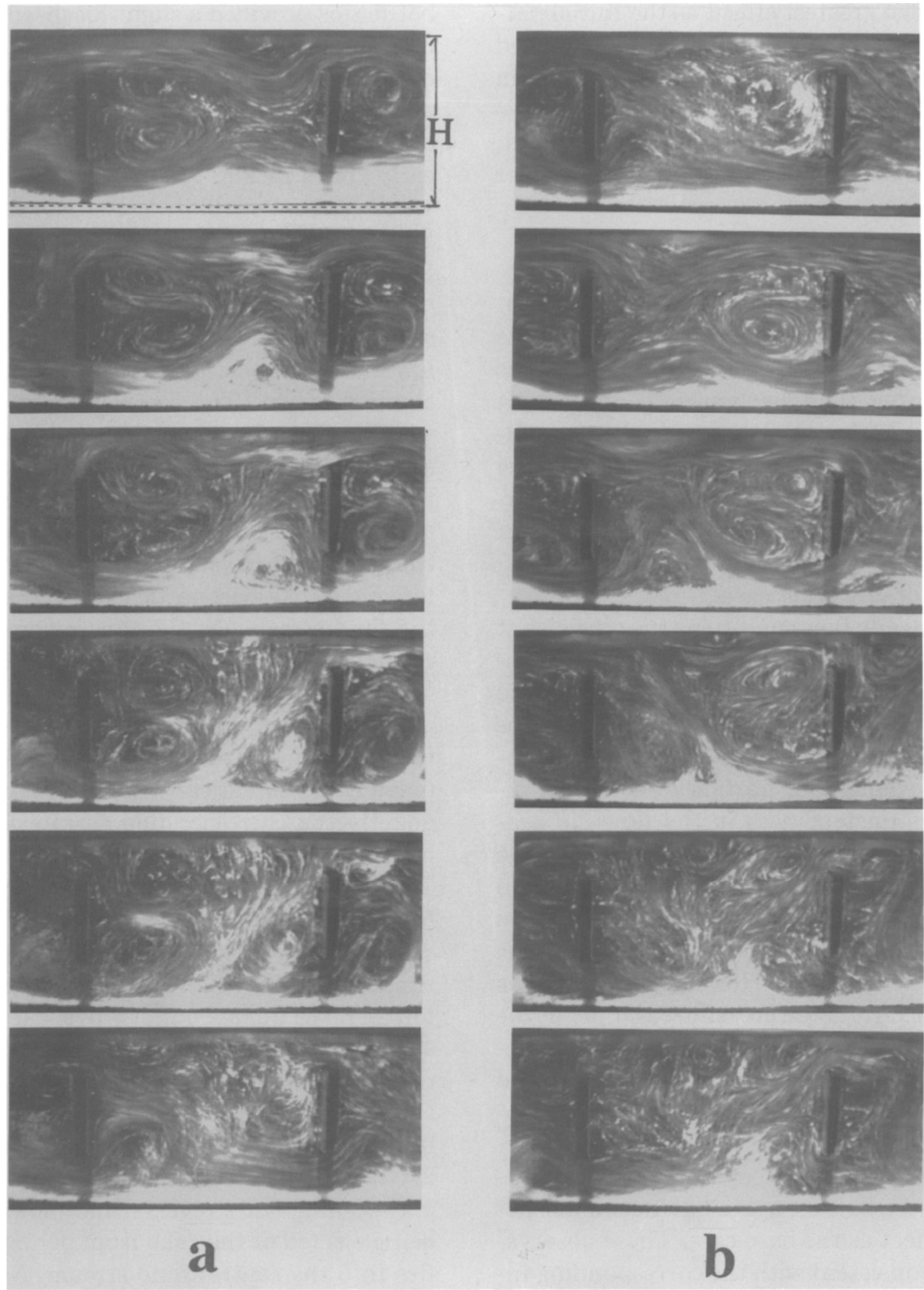

Fig. 9. Cake erosion in the centre baffled channel. $R e_{\mathrm{n}}=270$. (a) Flow accelerating from left to right, (b) reversed flow. 


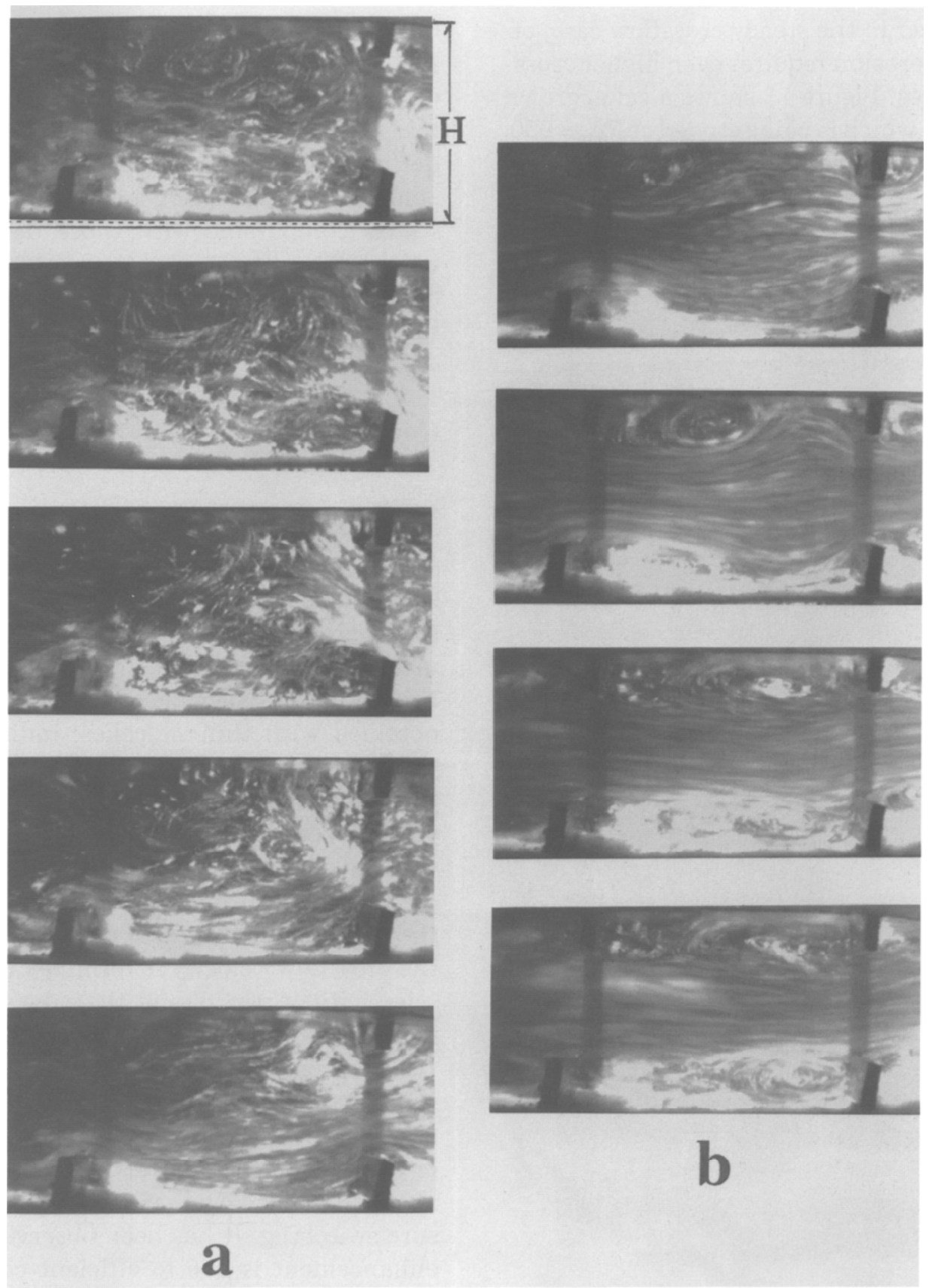

Fig. 10. Cake erosion in the wall baffled channel. $R e_{\mathrm{n}}=600$. (a) At flow reversal, (b) flow accelerating from left to right.

ticles into the mainstream provides a good cake erosion mechanism.

The essential difference between the erosion conditions for central and wall baffles shown in Figs. 9 and 10 is the net flow Reynolds number, i.e. 270 in the former case and 600 in the 
latter. Further in the steady crossflow case, effective cake erosion requires even higher crossflow velocities. Figure 11 shows a cake, grown at $R e_{\mathrm{n}}=270$, which is being eroded at $R e_{\mathrm{n}}=900$. Here the mechanism of removal is failure of the cake under the fluid shear rather than particle ejection by radial motion. Thus it can be seen that cake erosion can be attained in both steady and unsteady flow conditions but the central

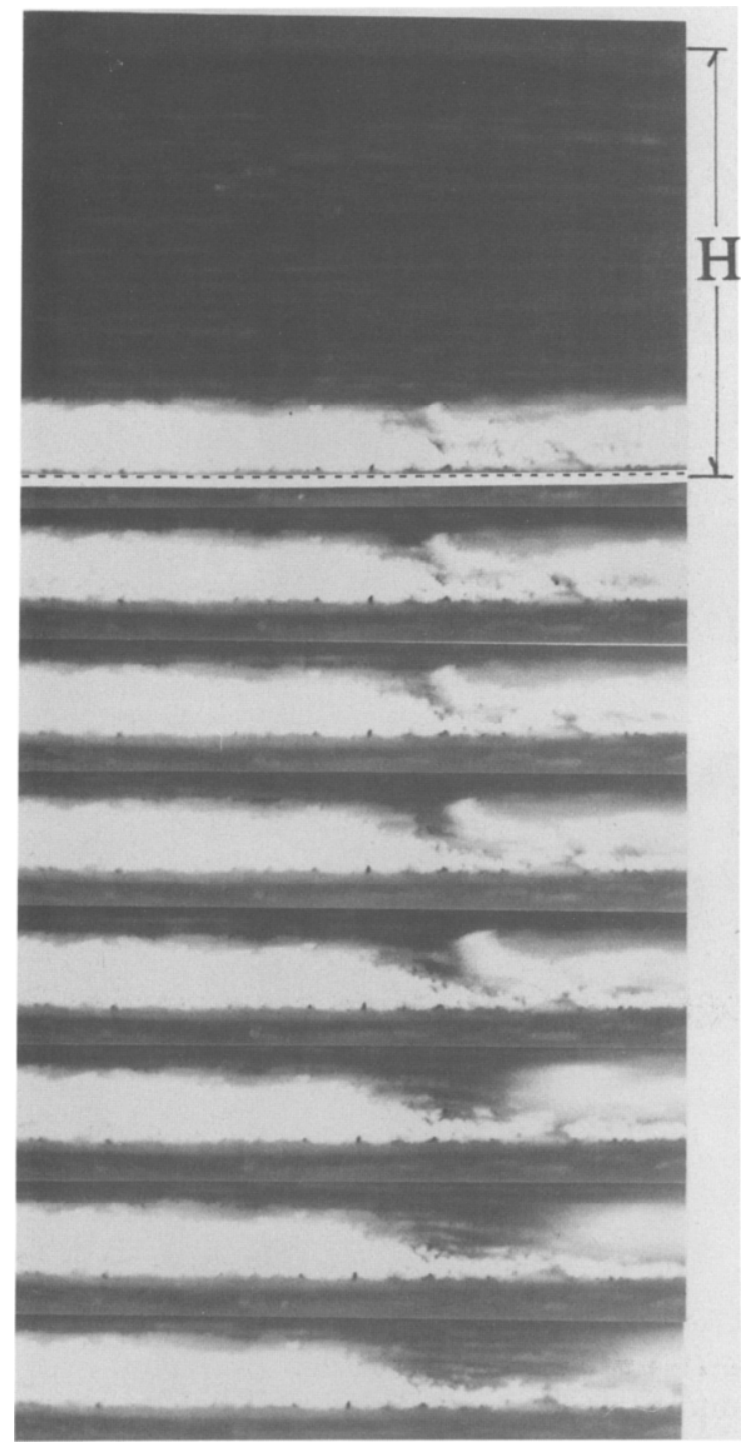

Fig. 11. Cake erosion at $R e_{\mathrm{n}}=900$. baffle geometry provides efficient removal at low $R e_{n}$, which may be important for the processing of shear sensitive material such as proteins [7] and suspension cultures [8]. The underlying fluid mechanics of the oscillatory flow configuration provides good radial mixing at $R e_{\mathrm{o}}$ values comparable with those demonstrated here $[6,9]$, making conditions suitable to grow and support shear sensitive cultures such as micro-organisms and animal cells [10].

\section{Conclusions}

It has been found that for the crossflow filtration of a neutrally buoyant particle suspension, increasing the crossflow velocity may lead to a decrease in the filtrate flux. Simultaneous measurements of the corresponding cake heights have shown that the lower fluxes are obtained with thinner cakes, indicating that these cakes offer a higher hydraulic resistance than thicker ones formed under low crossflow velocity conditions. This phenomenon can be explained in terms of the observed mechanisms for filter cake formation. It has been seen that the relative magnitude of the filtrate flux and the crossflow velocity determines the packing selectivity of particles at the cake surface and the conditions for limiting the cake height.

Under oscillatory crossflow conditions, the flux obtained from the system investigated can be dramatically enhanced by the introduction of centrally placed baffles within the channel above the filter, together with differential pressure switching. It has been observed that this enhancement is due to efficient cake erosion caused by eddy circulation both upstream and downstream of the baffles. It has also been found that effective cake erosion can be achieved using oscillatory flow with wall baffles and under steady flow conditions, but at the expense of increased crossflow velocity. 


\section{Acknowledgements}

The authors wish to thank the SERC and Schlumberger Cambridge Research for funding the project. We also wish to acknowledge work performed by Dave Freeman and Richard Lubienski during a postgraduate research project within the Dept. of Chemical Engincering at Cambridge, in which the effect of flux decline with increasing crossflow was first observed.

\section{References}

1 M.R. Hoogland, C.J.D. Fell, A.G. Fane and D.A.R. Jones, The optimum design of cross-flow filtration elements for mineral slurry processing, Proc. 5th World Filtration Congress, Nice, June 5-8, 1990, Société française de filtration, pp. 604-610.

2 G.B. Tanny, D. Hauk and U. Meuin, Biotechnical applications of a pleated crossflow microfiltration module, Desalination, 41 (1982) 299-312.

3 C.K. Colton, L.W. Henderson, C.A. Ford and M.J. Lysaght, Kinetics of hemodiafiltration. 1. In vitro transport characteristics of a hollow fibre blood ultrafilter, J. Lab. Clin. Med., 85 (1975) 355.
4 S.M. Finnigan and J.A. Howell, The effect of pulsatile flow on ultrafiltration fluxes in a baffled tubular membrane system, Chem. Eng. Res. Des., 67 (1989) 278280.

5 M.R. Mackley and N.E. Sherman, Cross-flow filtration mechanisms and kinetics, Chem. Eng. Sci., 47(12) (1992) 3067-3084.

6 C.R. Brunold, J.C.B. Hunns, M.R. Mackley and J.W. Thompson, Experimental observations on flow patterns and energy losses for oscillatory flow in ducts containing sharp edges, Chem. Eng. Sci., 44(5) (1989) 1227-1244.

7 M.R. Khan, D.E. Salt, D. Allen, M. Hoare and P. Dunnill, The effect of shear and associated factors on proteins in fermentation environment, Proc. IChemE Research Event, January 9-10, 1992, UMIST (1992) 287-289.

8 P.M. Kieran, D.M. Malone, P.F. MacLoughlin and G. Wilson, Investigation of the susceptibility of suspension cultures of Morinda citrifolia to hydrodynamic shear stress, Proc. IChemE Research Event, January 9-10, 1992, UMIST (1992) 232-234.

9 T. Howes, M.R. Mackley and E.P.L. Roberts, The simulation of chaotic mixing and dispersion for periodic flows in baffled channels, Chem. Eng. Sci., 47(7) (1991) 1669-1677.

10 S.T.L. Harrison and M.R. Mackley, A pulsatile flow bioreactor, Chem. Eng. Sci., 47(2) (1992) 490-493. 